\title{
ON EXPONENTIATION AND MULTIPLICATION OF $n$-ARY HYPERALGEBRAS
}

\author{
NITIMA CHAISANSUK
}

\begin{abstract}
In the paper, a new operation of product of $n$-ary hyperalgebras is introduced and studied which lies between their direct product and the direct product of their idempotent hulls. With respect to the product introduced, we give sufficient conditions for the validity of the first exponential law and a weak form of the second exponential law for a natural exponentiation of $n$-ary hyperalgebras.
\end{abstract}

\section{INTRODUCTION}

Birkhoff's direct arithmetic of partially ordered sets [1] was extended to relational systems by several authors, see, e.g., $[6,7]$ and $[9,10]$. Conversely, the cardinal arithmetic has been restricted from relational systems to ( $n$-ary) algebras in [11], to partial algebras in [13] and to hyperalgebras in [12]. In the present paper, we continue the study of the arithmetic of hyperalgebras from [12]. Note that, as for generality, hyperalgebras lie between relational systems and algebras because algebras are the relational systems that are both partial algebras and hyperalgebras.

In [7], M. Novotný and J. Šlapal introduced and studied a new operation of product of relational systems which is a combination of their direct sum and direct product. In this note, we restrict the operation of product discussed in [7] to hyperalgebras. We will study the behavior of the product obtained and will find conditions under which a naturally defined exponentiation of $n$-ary hyperalgebras satisfies the first exponential law with respect to the product.

The theory of hyperalgebras has intensively been studied since the beginning of 1990's (see [2]) when hyperalgebras proved to be useful for many application in various branches of mathematics and computer science (automata theory). The aim of the present paper is to contribute to further development of the theory of hyperalgebras. We will focus on the arithmetic of $n$-ary hyperalgebras. First, we will introduce the operation of the direct sum od $n$-ary hyperalgebras in a natural way and show that it is compatible with the direct product of the hyperalgebras in the sense that the direct product distributes over it. Then, we will introduce a new operation for $n$-ary hyperalgebras, called the combined product, and investigate its behavior. In particular, we will be interested in the relationship of the combined

$M S C$ (2010): primary 08A05, 08A99.

Keywords: $n$-ary hyperalgebra, diagonality, mediality, direct product, the first and second exponential laws.

This research was supported by the Royal Golden Jubilee Ph.D. Program of the Thailand Research Fund, the Graduate School and the Faculty of Science, Chiang Mai University, Thailand. 
product to the naturally defined exponentiation of $n$-ary hyperalgebras. We will find sufficient conditions for the validity of the first exponential law and a weak form of the second exponential law with respect to the combined product.

\section{Combined PRoduct of $n$-ARY Hyperalgebras}

Given sets $G, H$, we denote by $G^{H}$ the set of all mappings of $H$ into $G$. It is easy to see that there is a bijection $\varphi:\left(G^{H}\right)^{K} \rightarrow G^{H \times K}$ (where $\times$ denotes the cartesian product) given by $\varphi(h)(x, y)=h(y)(x)$ whenever $h \in\left(G^{H}\right)^{K}, x \in H$ and $y \in K$. The bijection $\varphi$ will be called canonical.

Throughout the paper, maps $f: G \rightarrow H$ ( $G, H$ sets) will often be denoted by indexed sets $\left(f_{i} ; i \in G\right)$ where $f_{i} \in H$ for every $i \in G$. Then, of course, $f_{i}$ means $f(i)$ for every $i \in G$.

Let $n$ be a non-negative integer. By an $n$-ary hyperalgebra we understand a pair $\mathbf{G}=(G, p)$ where $G$ is a non-empty set, called the carrier of $\mathbf{G}$, and $p: G^{n} \rightarrow \exp G \backslash\{\emptyset\}$ is a map, called the $n$-ary hyperoperation of $\mathbf{G}$. To avoid some unwanted singularities, we do not consider the trivial case $n=0$, i.e., we restrict our considerations to $n$-ary hyperalgebras with $n>0$ only. An $n$-ary hyperalgebra $\mathbf{G}=(G, p)$ with the property card $p\left(x_{1}, \ldots, x_{n}\right)=1$ for every $x_{1}, \ldots, x_{n} \in G$ is called an $n$-ary algebra. Binary hyperalgebras are usually called hypergroupoids.

Given an $n$-ary hyperalgebra $\mathbf{G}=(G, p)$ and non-empty subsets $X_{1}, \ldots, X_{n}$ of $G$, we put $p\left(X_{1}, \ldots, X_{n}\right)=\bigcup\left\{p\left(x_{1}, \ldots, x_{n}\right) ; x_{1} \in X_{1}, \ldots, x_{n} \in X_{n}\right\}$.

Let $\mathbf{G}=(G, p), \mathbf{H}=(H, q)$ be a pair of $n$-ary hyperalgebras. Then, $\mathbf{G}$ is called a subhyperalgebra of $\mathbf{H}$ provided that $G \subseteq H$ and $p\left(x_{1}, \ldots, x_{n}\right)=q\left(x_{1}, \ldots, x_{n}\right)$ whenever $x_{1}, \ldots x_{n} \in G$. Given $n$-ary hyperalgebras $\mathbf{G}, \mathbf{H}$ with the same carrier $G$, we put $\mathbf{G} \leq \mathbf{H}$ if $p\left(x_{1}, \ldots, x_{n}\right) \subseteq q\left(x_{1}, \ldots, x_{n}\right)$ whenever $x_{1}, \ldots x_{n} \in G$. A map $f: H \rightarrow G$ is called a homomorphism of $\mathbf{H}$ into $\mathbf{G}$ if $f\left(q\left(x_{1}, \ldots, x_{n}\right)\right) \subseteq$ $p\left(f\left(x_{1}\right), \ldots, f\left(x_{n}\right)\right)$. The set of all homomorphisms of $\mathbf{H}$ into $\mathbf{G}$ will be denoted by $\operatorname{Hom}(\mathbf{H}, \mathbf{G})$. If $f$ is a bijection of $H$ onto $G$ and $f\left(q\left(x_{1}, \ldots, x_{n}\right)\right)=$ $p\left(f\left(x_{1}\right), \ldots, f\left(x_{n}\right)\right)$ whenever $x_{1}, \ldots, x_{n} \in H$, then $f$ is called an isomorphism of $\mathbf{H}$ onto $\mathbf{G}$. In other words, an isomorphism of $\mathbf{H}$ onto $\mathbf{G}$ is a bijection $f: H \rightarrow G$ such that $f$ is a homomorphism of $\mathbf{H}$ into $\mathbf{G}$ and $f^{-1}$ is a homomorphism of $\mathbf{G}$ into $\mathbf{H}$. If there is an isomorphism of $\mathbf{H}$ onto $\mathbf{G}$, then we write $\mathbf{H} \cong \mathbf{G}$ and say that $\mathbf{H}$ and $\mathbf{G}$ areisomorphic. We say that $\mathbf{H}$ may be embedded into $\mathbf{G}$ and write $\mathbf{H} \preceq \mathbf{G}$ if there exists a subhyperalgebra $\mathbf{G}^{\prime}$ of $\mathbf{G}$ such that $\mathbf{H} \cong \mathbf{G}^{\prime}$.

The direct product of a non-empty family $\mathbf{G}_{i}=\left(G_{i}, p_{i}\right), i \in I$, of $n$-ary hyperalgebras is the $n$-ary hyperalgebra $\prod_{i \in I} \mathbf{G}_{i}=\left(\prod_{i \in I} G_{i}, q\right)$ where $\prod_{i \in I} G_{i}$ is the cartesian product and $q$ is given by $q\left(f_{1}, \ldots, f_{n}\right)=\prod_{i \in I} p_{i}\left(f_{1}(i), \ldots, f_{n}(i)\right)$ whenever $f_{1}, \ldots, f_{n} \in \prod_{i \in I} G_{i}$. If the set $I$ is finite, say $I=\{1, \ldots, m\}$, then we write $\mathbf{G}_{1} \times \ldots \times \mathbf{G}_{m}$ instead of $\prod_{i \in I} G_{i}$. If $\mathbf{G}_{i}=\mathbf{G}$ for every $i \in I$, then we write $\mathbf{G}^{I}$ instead of $\prod_{i \in I} \mathbf{G}_{i}$.

The direct sum of a family $\mathbf{G}_{i}=\left(G, p_{i}\right), i \in I$, of $n$-ary hyperalgebras is the $n$ ary hyperalgebra $\sum_{i \in I} \mathbf{G}_{i}=(G, q)$ where, for every $x_{1}, \ldots, x_{n} \in G, q\left(x_{1}, \ldots, x_{n}\right)$ $=\bigcup_{i \in I} p_{i}\left(x_{1}, \ldots, x_{n}\right)$. If the set $I$ is finite, say $I=\{1, \ldots, m\}$, then we write $\mathbf{G}_{1} \uplus \ldots \uplus \mathbf{G}_{m}$ instead of $\sum_{i \in I} \mathbf{G}_{i}$.

An $n$-ary hyperalgebra $\mathbf{G}=(G, p)$ is said to be idempotent if, for every $x \in G$, $x \in p\left(x_{1}, \ldots, x_{n}\right)$ whenever $x=x_{1}=\ldots=x_{n}$. Let $\mathbf{G}=(G, p)$ be an $n$-ary 
hyperalgebra. For every $x_{1}, \ldots, x_{n} \in G$, we put $\bar{p}\left(x_{1}, \ldots, x_{n}\right)= \begin{cases}p\left(x_{1}, \ldots, x_{n}\right) \cup\{x\} & \text { if there is } x \in G \text { with } x=x_{1}=\ldots=x_{n}, \\ p\left(x_{1}, \ldots, x_{n}\right) & \text { if there are } i, j \in\{1, \ldots, n\} \text { with } x_{i} \neq x_{j} .\end{cases}$

The $n$-ary hyperalgebra $(G, \bar{p})$ is called the idempotent hull of $\mathbf{G}$ and is denoted by $\overline{\mathbf{G}}$.

Let $\mathbf{G}_{i}=\left(G_{i}, p_{i}\right), i \in I$, be a non-empty family of $n$-ary hyperalgebras. The combined product of the family $\mathbf{G}_{i}, i \in I$, is the $n$-ary hyperalgebra $\otimes_{i \in I} \mathbf{G}_{i}=$ $\left(\prod_{i \in I} G_{i}, r\right)$ given by $\bigotimes_{i \in I} \mathbf{G}_{i}=\sum_{i \in I} \prod_{j \in I} \mathbf{G}_{i j}$ where

$$
\mathbf{G}_{i j}= \begin{cases}\overline{\mathbf{G}}_{j} & \text { if } i=j, \\ \mathbf{G}_{j} & \text { if } i \neq j .\end{cases}
$$

Thus, for any $\left(x_{1}^{i} ; i \in I\right), \ldots,\left(x_{n}^{i} ; i \in I\right) \in \prod_{i \in I} \mathbf{G}_{i}$, we have $\left(x^{i} ; i \in I\right) \in$ $r\left(\left(x_{1}^{i} ; i \in I\right), \ldots,\left(x_{n}^{i} ; i \in I\right)\right)$ if and only if there exists a subset $J \subseteq I$, card $J \leq 1$, such that $x^{i} \in p_{i}\left(x_{1}^{i}, \ldots, x_{n}^{i}\right)$ for every $i \in I \backslash J$ and $x^{i}=x_{1}^{i}=x_{2}^{i}=\ldots=x_{n}^{i}$ and for every $i \in J$.

If the set $I$ is finite, say $I=\{1, \ldots, m\}$, we write $\mathbf{G}_{1} \otimes \ldots \otimes \mathbf{G}_{m}$ instead of $\bigotimes_{i \in I} \mathbf{G}_{i}$. We then clearly have $\mathbf{G}_{1} \otimes \ldots \otimes \mathbf{G}_{m}=\left(\mathbf{G}_{11} \times \mathbf{G}_{12} \times \ldots \times \mathbf{G}_{1 m}\right) \uplus\left(\mathbf{G}_{21} \times\right.$ $\left.\mathbf{G}_{22} \times \ldots \times \mathbf{G}_{2 m}\right) \uplus \ldots \uplus\left(\mathbf{G}_{m 1} \times \mathbf{G}_{m 2} \ldots, \times \mathbf{G}_{m m}\right)=\left(\overline{\mathbf{G}}_{1} \times \mathbf{G}_{2} \times \ldots \times \mathbf{G}_{m}\right) \uplus$ $\left(\mathbf{G}_{1} \times \overline{\mathbf{G}}_{2} \times \ldots \times \mathbf{G}_{m}\right) \uplus \ldots \uplus\left(\mathbf{G}_{1} \times \mathbf{G}_{2} \times \ldots \times \overline{\mathbf{G}}_{m}\right)$. In particular, if $I=\{1,2\}$, then, for any $\left(x_{1}, y_{1}\right), \ldots,\left(x_{n}, y_{n}\right) \in \mathbf{G}_{1} \times \mathbf{G}_{2},(x, y) \in r\left(\left(x_{1}, y_{1}\right), \ldots,\left(x_{n}, y_{n}\right)\right)$ if and only if one of the following three conditions is satisfied:

(i) $x \in p_{1}\left(x_{1}, \ldots, x_{n}\right)$ and $y \in p_{2}\left(y_{1}, \ldots, y_{n}\right)$,

(ii) $x=x_{1}=x_{2}=\ldots=x_{n}$ and $y \in p_{2}\left(y_{1}, \ldots, y_{n}\right)$,

(iii) $x \in p_{1}\left(x_{1}, \ldots, x_{n}\right)$ and $y=y_{1}=y_{2}=\ldots=y_{n}$.

If $I$ is a singleton, say $I=\{j\}$, then $\bigotimes_{i \in I} \mathbf{G}_{i}=\overline{\mathbf{G}}_{j}$.

Remark 2.1. Let $\mathbf{G}_{i}, i \in I$ be a family of $n$-ary hyperalgebras. We clearly have $\prod_{i \in I} \mathbf{G}_{i} \leq \bigotimes_{i \in I} \mathbf{G}_{i} \leq \prod_{i \in I} \overline{\mathbf{G}}_{i}=\bigotimes_{i \in I} \overline{\mathbf{G}}_{i}$ and $\overline{\bigotimes_{i \in I} \mathbf{G}_{i}} \leq \bigotimes_{i \in I} \overline{\mathbf{G}}_{i}$. If $\mathbf{G}_{i}$ is idempotent for every $i \in I$, then all the previous inequalities become equalities. If at most one of the $n$-ary hyperalgebras $\mathbf{G}_{i}, i \in I$, is not idempotent, then $\bigotimes_{i \in I} \mathbf{G}_{i}$ is idempotent.

The following statement shows that the direct sum of $n$-ary hyperalgebras is compatible with their direct product in the sense that the direct product distributes over it.

Theorem 2.2. Let $\mathbf{G}_{i}=\left(G_{i}, p_{i}\right), i \in I$, be a non-empty family of $n$-ary hyperalgebras and let $\mathbf{H}=(H, q)$ be an n-ary hyperalgebra. Then $\sum_{i \in I} \mathbf{H} \otimes \mathbf{G}_{i}=$ $\mathbf{H} \otimes \sum_{i \in I} \mathbf{G}_{i}$.

Proof. Let $\sum_{i \in I} \mathbf{G}_{i}=(G, s), \mathbf{H} \otimes \mathbf{G}_{i}=\left(H \times G, r_{i}\right)$ for each $i \in I, \mathbf{H} \otimes$ $\sum_{i \in I} \mathbf{G}_{i}=(H \times G, u)$ and $\sum_{i \in I} \mathbf{H} \otimes \mathbf{G}_{i}=(H \times G, v)$. We will show that $u\left(\left(x_{1}, y_{1}\right), \ldots,\left(x_{n}, y_{n}\right)\right)=v\left(\left(x_{1}, y_{1}\right), \ldots,\left(x_{n}, y_{n}\right)\right)$ for every $\left(x_{1}, y_{1}\right), \ldots,\left(x_{n}, y_{n}\right) \in$ $H \times G$. It is easy to see that the following five conditions are equivalent:

(a) $(x, y) \in u\left(\left(x_{1}, y_{1}\right), \ldots,\left(x_{n}, y_{n}\right)\right)$;

(b) one of the following three cases occurs:

(i) $x \in q\left(x_{1}, \ldots, x_{n}\right)$ and $y \in s\left(y_{1}, \ldots, y_{n}\right)$, 
(ii) $x=x_{1}=x_{2}=\ldots=x_{n}$ and $y \in s\left(y_{1}, \ldots, y_{n}\right)$,

(iii) $x \in q\left(x_{1}, \ldots, x_{n}\right)$ and $y=y_{1}=y_{2}=\ldots=y_{n}$;

(c) one of the following three cases occurs:

(i) $x \in q\left(x_{1}, \ldots, x_{n}\right)$ and $y \in p_{i}\left(y_{1}, \ldots, y_{n}\right)$ for some $i \in I$,

(ii) $x=x_{1}=x_{2}=\ldots=x_{n}$ and $y \in p_{i}\left(y_{1}, \ldots, y_{n}\right)$ for some $i \in I$,

(iii) $x \in q\left(x_{1}, \ldots, x_{n}\right)$ and $y=y_{1}=y_{2}=\ldots=y_{n}$;

(d) $(x, y) \in r_{i}\left(\left(x_{1}, y_{1}\right), \ldots,\left(x_{n}, y_{n}\right)\right)$ for some $i \in I$;

(e) $(x, y) \in v\left(\left(x_{1}, y_{1}\right), \ldots,\left(x_{n}, y_{n}\right)\right)$.

This proves the statement.

\section{POWER OF hyperalgebras}

Definition 3.1. An $n$-ary hyperalgebra $(G, p)$ is called medial if, for every $n \times n$-matrix $\left(a_{i j}\right)$ over $G$, from $x_{i} \in p\left(a_{i 1}, \ldots, a_{i n}\right)$ for each $i=1, \ldots, n$ and $y_{j} \in$ $p\left(a_{1 j}, \ldots, a_{n j}\right)$ for each $j=1, \ldots, n$, it follows that $p\left(x_{1}, \ldots, x_{n}\right)=p\left(y_{1}, \ldots, y_{n}\right)$.

The medial $n$-ary algebras are studied in [11]. The mediality may be extended, in a natural way, from $n$-ary algebras to universal algebras. The medial universal algebras are then often called commutative or the universal algebras satisfying the interchange law and are dealt with, e.g., in $[4,5]$ and [11]. The medial groupoids are studied in [3].

Example 3.2. (1) Let $(X, \leq)$ be a partially ordered set with a least element 0 and let $A$ be the set of all atoms of $(X, \leq)$. Put $0^{\prime}=\{0\}$ and, for any $x \in X$ with $x \neq 0$, put $x^{\prime}=\{y \in X ; y<x$ and $y \in A \cup\{0\}\}$. Further, for any pair $x, y \in X$, put $x * y=x^{\prime} \cap y^{\prime}$. Then, $(X, *)$ is a medial hypergroupoid. Indeed, it can easily be seen that, for any $a, b, c, d \in X$, we have $x * y=f * g$ whenever $x \in a * b, y \in c * d$, $f \in a * c$ and $g \in b * d$.

(2) Every unary algebra $\mathbf{G}$ consisting of two-element cycles only is medial and, moreover, the $n$-ary hyperalgebra $\overline{\mathbf{G}}$ is medial, too.

Lemma 3.3. Let $\mathbf{H}=(H, q)$ and $\mathbf{G}=(G, p)$ be $n$-ary hyperalgebras and let $f_{1}, \ldots, f_{n} \in \operatorname{Hom}(\mathbf{H}, \mathbf{G})$. Let $f: H \rightarrow G$ be a map such that $f(x) \in$ $p\left(f_{1}(x), \ldots, f_{n}(x)\right)$ for every $x \in H$. If $\mathbf{G}$ is medial, then $f$ is a homomorphism from $\mathbf{H}$ into $\mathbf{G}$.

Proof. Let $x_{1}, \ldots, x_{n} \in H$. We will show that $f\left(q\left(x_{1}, \ldots, x_{n}\right)\right) \subseteq p\left(f\left(x_{1}\right), \ldots\right.$, $\left.f\left(x_{n}\right)\right)$. Let $y \in f\left(q\left(x_{1}, \ldots, x_{n}\right)\right)$. Then, there exists $x \in q\left(x_{1}, \ldots, x_{n}\right)$ such that $y=f(x)$, so that $f(x) \in f\left(q\left(x_{1}, \ldots, x_{n}\right)\right)$. Since $f_{1}, \ldots, f_{n} \in \operatorname{Hom}(\mathbf{H}, \mathbf{G})$ and $x \in$ $q\left(x_{1}, \ldots, x_{n}\right)$, we have $f_{j}(x) \in f_{j}\left(q\left(x_{1}, \ldots, x_{n}\right)\right) \subseteq p\left(f_{j}\left(x_{1}\right), \ldots, f_{j}\left(x_{n}\right)\right)$ for each $j=1, \ldots, n$. By the assumption, we have $f\left(x_{i}\right) \in p\left(f_{1}\left(x_{i}\right), \ldots, f_{n}\left(x_{i}\right)\right)$ for each $i=$ $1, \ldots, n$ and $f(x) \in p\left(f_{1}(x), \ldots, f_{n}(x)\right)$. Since $\mathbf{G}$ is medial, $p\left(f_{1}(x), \ldots, f_{n}(x)\right)=$ $p\left(f\left(x_{1}\right), \ldots, f\left(x_{n}\right)\right)$. Thus, $y=f(x) \in p\left(f\left(x_{1}\right), \ldots, f\left(x_{n}\right)\right)$.

The previous lemma enables us to define:

Definition 3.4. Let $\mathbf{G}=(G, p)$ and $\mathbf{H}=(H, q)$ be $n$-ary hyperalgebras and let $\mathbf{G}$ be medial. The power of $\mathbf{G}$ and $\mathbf{H}$ is the $n$-ary hyperalgebra $\mathbf{G}^{\mathbf{H}}=$ $(\operatorname{Hom}(\mathbf{H}, \mathbf{G}), r)$ where, for any $f_{1}, \ldots, f_{n} \in \operatorname{Hom}(\mathbf{H}, \mathbf{G}), r\left(f_{1}, \ldots, f_{n}\right)=\{f \in$ $G^{H} ; f(x) \in p\left(f_{1}(x), \ldots, f_{n}(x)\right)$ for each $\left.x \in H\right\}$. 
It is easy to see that the power $\mathbf{G}^{\mathbf{H}}$ of $n$-ary hyperalgebras is idempotent whenever $\mathbf{G}$ is idempotent (and medial). $\mathbf{G}^{\mathbf{H}}$.

Theorem 3.5. Let $\mathbf{G}, \mathbf{H}$ be n-ary hyperalgebras. If $\mathbf{G}$ is medial, then so is

Proof. Let $\mathbf{G}=(G, p), \mathbf{H}=(H, q), \mathbf{G}^{\mathbf{H}}=(\operatorname{Hom}(\mathbf{H}, \mathbf{G}), r)$ and let $\left(f_{i j}\right)$ be an $n \times n$-matrix over $\operatorname{Hom}(\mathbf{H}, \mathbf{G})$. Suppose $h_{i} \in r\left(f_{i 1}, \ldots, f_{i n}\right)$ for all $i=1, \ldots, n$ and $g_{j} \in r\left(f_{1 j}, \ldots, f_{n j}\right)$ for all $j=1, \ldots, n$. For every $x \in H$, we have $h_{i}(x) \in$ $p\left(f_{i 1}(x), \ldots, f_{i n}(x)\right)$ for all $i=1, \ldots, n$ and $g_{j}(x) \in p\left(f_{1 j}(x), \ldots, f_{n j}(x)\right)$ for all $j=$ $1, \ldots, n$. Since $\mathbf{G}$ is medial, we have $p\left(h_{1}(x), \ldots, h_{n}(x)\right)=p\left(g_{1}(x), \ldots, g_{n}(x)\right)$ for every $x \in H$. Therefore, $r\left(h_{1}, \ldots, h_{n}\right)=r\left(g_{1}, \ldots, g_{n}\right)$, so that $\mathbf{G}^{\mathbf{H}}$ is medial.

Definition 3.6. An $n$-ary hyperalgebra $(G, p)$ is called diagonal if, for every $n \times n$-matrix $\left(a_{i j}\right)$ over $G$, we have $p\left(p\left(a_{11}, \ldots, a_{1 n}\right), \ldots, p\left(a_{n 1}, \ldots, a_{n n}\right)\right) \cap$ $p\left(p\left(a_{11}, \ldots, a_{n 1}\right), \ldots, p\left(a_{1 n}, \ldots, a_{n n}\right)\right) \subseteq p\left(a_{11}, \ldots, a_{n n}\right)$.

For idempotent algebras, the diagonality introduced coincides with the diagonality studied in [8].

Example 3.7. It may easily be seen that the hypergroupoid from Example 3.2 is diagonal.

Theorem 3.8. Let $\mathbf{G}, \mathbf{H}$ be n-ary hyperalgebras. If $\mathbf{G}$ is medial and diagonal, then $\mathbf{G}^{\mathbf{H}}$ is diagonal.

Proof. Let $\mathbf{G}=(G, p), \mathbf{H}=(H, q), \mathbf{G}^{\mathbf{H}}=(\operatorname{Hom}(\mathbf{H}, \mathbf{G}), r)$ and let $\left(f_{i j}\right)$ be an $n \times n$-matrix over $\operatorname{Hom}(\mathbf{H}, \mathbf{G})$. Suppose that $f \in r\left(r\left(f_{11}, \ldots, f_{1 n}\right), \ldots, r\left(f_{n 1}, \ldots\right.\right.$, $\left.\left.f_{n n}\right)\right) \cap r\left(r\left(f_{11}, \ldots, f_{n 1}\right), \ldots, r\left(f_{1 n}, \ldots, f_{n n}\right)\right)$. Then, $f(x) \in p\left(p\left(f_{11}(x), \ldots\right.\right.$, $\left.\left.f_{1 n}(x)\right), \ldots, r\left(f_{n 1}(x), \ldots, f_{n n}(x)\right)\right) \cap p\left(p\left(f_{11}(x), \ldots, f_{n 1}(x)\right), \ldots, p\left(f_{1 n}(x), \ldots\right.\right.$, $\left.\left.f_{n n}(x)\right)\right)$ for every $x \in H$. Since $\mathbf{G}$ is diagonal, we have $f(x) \in p\left(f_{11}(x), \ldots\right.$, $\left.f_{n n}(x)\right)$ for every $x \in H$. Thus, $f \in r\left(f_{11}, \ldots, f_{n n}\right)$. Hence, $\mathbf{G}^{\mathbf{H}}$ is diagonal.

We will find sufficient conditions for the validity of the first exponential law for exponentiation of $n$-ary hyperalgebras with respect to the combined product, i.e., the law

$$
\left(\mathbf{G}^{\mathbf{H}}\right)^{\mathbf{K}} \cong \mathbf{G}^{\mathbf{H} \otimes \mathbf{K}} .
$$

Lemma 3.9. Let $\mathbf{G}, \mathbf{H}, \mathbf{K}$ be n-ary hyperalgebras and let $\mathbf{G}$ be medial and diagonal. Then, the canonical bijection $\varphi:\left(G^{H}\right)^{K} \rightarrow G^{H \times K}$ restricted to $\operatorname{Hom}\left(\mathbf{K}, \mathbf{G}^{\mathbf{H}}\right)$ is a bijection of $\operatorname{Hom}\left(\mathbf{K}, \mathbf{G}^{\mathbf{H}}\right)$ onto $\operatorname{Hom}(\mathbf{H} \otimes \mathbf{K}, \mathbf{G})$.

Proof. Let $\mathbf{G}=(G, p), \mathbf{H}=(H, q), \mathbf{K}=(K, s), \mathbf{G}^{\mathbf{H}}=(\operatorname{Hom}(\mathbf{H}, \mathbf{G}), r), \mathbf{H} \otimes$ $\mathbf{K}=(H \times K, v)$ and let $\mathbf{G}$ be diagonal and medial.

Let $h \in \operatorname{Hom}\left(\mathbf{K}, \mathbf{G}^{\mathbf{H}}\right)$ and let $\left(y_{1}, z_{1}\right), \ldots,\left(y_{n}, z_{n}\right) \in H \times K$. To show that $\varphi(h)\left(v\left(\left(y_{1}, z_{1}\right), \ldots,\left(y_{n}, z_{n}\right)\right) \subseteq p\left(\varphi(h)\left(y_{1}, z_{1}\right), \ldots, \varphi(h)\left(y_{n}, z_{n}\right)\right)\right.$, let $x \in \varphi(h)$ $\left(v\left(\left(y_{1}, z_{1}\right), \ldots,\left(y_{n}, z_{n}\right)\right)\right)$. Then, there exists $(y, z) \in v\left(\left(y_{1}, z_{1}\right), \ldots,\left(y_{n}, z_{n}\right)\right)$ such that $x=\varphi(h)(y, z)$. Thus, one of the following three conditions is satisfied:

(i) $y \in q\left(y_{1}, \ldots, y_{n}\right)$ and $z \in s\left(z_{1}, \ldots, z_{n}\right)$,

(ii) $y=y_{1}=y_{2}=\ldots=y_{n}$ and $z \in s\left(z_{1}, \ldots, z_{n}\right)$,

(iii) $y \in q\left(y_{1}, \ldots, y_{n}\right)$ and $z=z_{1}=z_{2}=\ldots=z_{n}$. 
If $z \in s\left(z_{1}, \ldots, z_{n}\right)$, then $h(z) \in h\left(s\left(z_{1}, \ldots, z_{n}\right)\right) \subseteq r\left(h\left(z_{1}\right), \ldots, h\left(z_{n}\right)\right)$, hence $\varphi(h)(y, z)=h(z)(y) \in p\left(\left(h\left(z_{1}\right)(y), \ldots, h\left(z_{n}\right)(y)\right)=p\left(\varphi(h)\left(y, z_{1}\right), \ldots, \varphi(h)\left(y, z_{n}\right)\right.\right.$ for every $y \in H$. Thus, $\varphi(h)(y, z) \in p\left(\varphi(h)\left(y_{1}, z_{1}\right), \ldots, \varphi(h)\left(y_{n}, z_{n}\right)\right)$ provided that (ii) is satisfied.

If $y \in q\left(y_{1}, \ldots, y_{n}\right)$, then $\varphi(h)(y, z)=h(z)(y) \in h(z)\left(q\left(y_{1}, \ldots, y_{n}\right)\right) \subseteq p(h(z)$ $\left.\left(y_{1}\right), \ldots, h(z)\left(y_{n}\right)\right)=p\left(\varphi(h)\left(y_{1}, z\right), \ldots, \varphi(h)\left(y_{n}, z\right)\right)$ for every $z \in K$. Thus, $\varphi(h)(y, z) \in p\left(\varphi(h)\left(y_{1}, z_{1}\right), \ldots, \varphi(h)\left(y_{n}, z_{n}\right)\right)$ provided that (iii) is satisfied.

Suppose that (i) is satisfied. Since $z \in s\left(z_{1}, \ldots, z_{n}\right)$, by the above considerations, we have $\varphi(h)\left(y_{i}, z\right) \in p\left(\varphi(h)\left(y_{i}, z_{1}\right), \ldots, \varphi(h)\left(y_{i}, z_{n}\right)\right)$ for every $i=1, \ldots, n$ and $\varphi(h)(y, z) \in p\left(\varphi(h)\left(y, z_{1}\right), \ldots, \varphi(h)\left(y, z_{n}\right)\right)$. Since $y \in q\left(y_{1}, \ldots, y_{n}\right)$, by the above considerations we have $\varphi(h)\left(y, z_{j}\right) \in p\left(\varphi(h)\left(y_{1}, z_{j}\right), \ldots, \varphi(h)\left(y_{n}, z_{j}\right)\right)$ for every $j=1, \ldots, n$ and $\varphi(h)(y, z) \in p\left(\varphi(h)\left(y_{1}, z\right), \ldots, \varphi(h)\left(y_{n}, z\right)\right)$. The diagonality of $\mathbf{G}$ implies $\varphi(h)(y, z) \in p\left(\varphi(h)\left(y_{1}, z_{1}\right), \ldots, \varphi(h)\left(y_{n}, z_{n}\right)\right)$. We have shown that $\varphi(h) \in \operatorname{Hom}(\mathbf{H} \otimes \mathbf{K}, \mathbf{G})$.

Let $g \in \operatorname{Hom}(\mathbf{H} \otimes \mathbf{K}, \mathbf{G}), z_{1}, \ldots, z_{n} \in K$. To show that $\varphi^{-1}(g)\left(s\left(z_{1}, \ldots, z_{n}\right)\right)$ $\subseteq r\left(\varphi^{-1}(g)\left(z_{1}\right), \ldots, \varphi^{-1}(g)\left(z_{n}\right)\right)$, let $x \in \varphi^{-1}(g)\left(s\left(z_{1}, \ldots, z_{n}\right)\right)$. Then, there exists $z \in s\left(z_{1}, \ldots, z_{n}\right)$ such that $x \in \varphi^{-1}(g)(z)$. Let $y \in H$. Thus, $(y, z) \in$ $v\left(\left(y, z_{1}\right), \ldots,\left(y, z_{n}\right)\right)$. Since $g \in \operatorname{Hom}(\mathbf{H} \otimes \mathbf{K}, \mathbf{G})$, we have $g(y, z) \in g\left(v\left(\left(y, z_{1}\right)\right.\right.$ $\left., \ldots,\left(y, z_{n}\right)\right) \subseteq p\left(g\left(y, z_{1}\right), \ldots, g\left(y, z_{n}\right)\right)$. It follows that $\varphi^{-1}(g)(z)(y) \in p\left(\varphi^{-1}(g)\right.$ $\left.\left(z_{1}\right)(y), \ldots, \varphi^{-1}(g)\left(z_{n}\right)(y)\right)$, hence $\varphi^{-1}(g)(z) \in p\left(\varphi^{-1}(g)\left(z_{1}\right), \ldots, \varphi^{-1}(g)\left(z_{n}\right)\right)$. Therefore, $\varphi^{-1}(g)\left(s\left(z_{1}, \ldots, z_{n}\right)\right) \subseteq r\left(\varphi^{-1}(g)\left(z_{1}\right), \ldots, \varphi^{-1}(g)\left(z_{n}\right)\right)$. Consequently, $\varphi^{-1}(g) \in \operatorname{Hom}\left(\mathbf{K}, \mathbf{G}^{\mathbf{H}}\right)$.

Theorem 3.10. Let $\mathbf{G}, \mathbf{H}, \mathbf{K}$ be n-ary hyperalgebras. If $\mathbf{G}$ is medial and diagonal, then

$$
\left(\mathbf{G}^{\mathbf{H}}\right)^{\mathbf{K}} \cong \mathbf{G}^{\mathbf{H} \otimes \mathbf{K}} .
$$

Proof. Let $\mathbf{G}=(G, p), \mathbf{H}=(H, q), \mathbf{K}=(K, s), \mathbf{G}^{\mathbf{H}}=(H o m(\mathbf{H}, \mathbf{G}), r), \mathbf{H} \otimes$ $\mathbf{K}=(H \times K, v),\left(\mathbf{G}^{\mathbf{H}}\right)^{\mathbf{K}}=\left(\operatorname{Hom}\left(\mathbf{K}, \mathbf{G}^{\mathbf{H}}\right), t\right), \mathbf{G}^{\mathbf{H} \otimes \mathbf{K}}=(\operatorname{Hom}(\mathbf{H} \otimes \mathbf{K}, \mathbf{G}), u)$ and let $\mathbf{G}$ be diagonal and medial. Because of Lemma 3.9, we are to show that $\varphi\left(t\left(h_{1}, \ldots, h_{n}\right)\right)=u\left(\varphi\left(h_{1}\right), \ldots, \varphi\left(h_{n}\right)\right)$ whenever $h_{1}, \ldots, h_{n} \in \operatorname{Hom}\left(\mathbf{K}, \mathbf{G}^{\mathbf{H}}\right)$.

Let $x \in \varphi\left(t\left(h_{1}, \ldots, h_{n}\right)\right)$. Then, there exists $h \in t\left(h_{1}, \ldots, h_{n}\right)$ such that $x=$ $\varphi(h)$. Since $h \in t\left(h_{1}, \ldots, h_{n}\right)$, we have $h(z) \in r\left(h_{1}(z), \ldots, h_{n}(z)\right)$ for every $z \in K$. Therefore, $h(z)(y) \in p\left(h_{1}(z)(y), \ldots, h_{n}(z)(y)\right)$ for every $y \in H$. Thus $\varphi(h)(y, z) \in$ $p\left(\varphi\left(h_{1}\right)(y, z), \ldots, \varphi\left(h_{n}\right)(y, z)\right)$ for every $(y, z) \in H \times K$. Hence, $\varphi(h) \in u\left(\varphi\left(h_{1}\right)\right.$, $\left.\ldots, \varphi\left(h_{n}\right)\right)$. Consequently, $\varphi\left(t\left(h_{1}, \ldots, h_{n}\right)\right) \subseteq u\left(\varphi\left(h_{1}\right), \ldots, \varphi\left(h_{n}\right)\right)$.

Conversely, let $x \in u\left(\varphi\left(h_{1}\right), \ldots, \varphi\left(h_{n}\right)\right)$. By Lemma 3.9, there exists $h \in$ $\operatorname{Hom}\left(\mathbf{K}, \mathbf{G}^{\mathbf{H}}\right)$ with $x=\varphi(h)$, so that $\varphi(h) \in u\left(\varphi\left(h_{1}\right), \ldots, \varphi\left(h_{n}\right)\right)$. Then, $\varphi(h)(y, z)$ $\in p\left(\varphi\left(h_{1}\right)(y, z), \ldots, \varphi\left(h_{n}\right)(y, z)\right)$ for every $(y, z) \in H \times K$, which implies $h(z)(y) \in$ $p\left(h_{1}(z)(y), \ldots, h_{n}(z)(y)\right)$ for every $y \in H$ and $z \in K$. It follows that $h(z) \in$ $r\left(h_{1}(z), \ldots, h_{n}(z)\right)$ for every $z \in K$. Therefore, $h \in t\left(h_{1}, \ldots, h_{n}\right)$, thus $\varphi(h) \in$ $\varphi\left(t\left(h_{1}, \ldots, h_{n}\right)\right)$. Hence, $u\left(\varphi\left(h_{1}\right), \ldots, \varphi\left(h_{n}\right)\right) \subseteq \varphi\left(t\left(h_{1}, \ldots, h_{n}\right)\right)$.

We have shown that $\varphi\left(t\left(h_{1}, \ldots, h_{n}\right)\right)=u\left(\varphi\left(h_{1}\right), \ldots, \varphi\left(h_{n}\right)\right)$. Thus, $\left(\mathbf{G}^{\mathbf{H}}\right)^{\mathbf{K}}$ is isomorphic to $\mathbf{G}^{\mathbf{H} \otimes \mathbf{K}}$.

Remark 3.11. It follows from Theorem 3.10 that the first exponential law with respect to the direct product, i.e., the law $\left(\mathbf{G}^{\mathbf{H}}\right)^{\mathbf{K}} \cong \mathbf{G}^{\mathbf{H} \times \mathbf{K}}$, is valid whenever $\mathbf{G}, \mathbf{H}, \mathbf{K}$ are $n$-ary hyperalgebras, $\mathbf{G}$ medial and diagonal and $\mathbf{H}, \mathbf{K}$ idempotent. 
This result was proved in [12]. But Theorem 3.10 says that, with respect to the combined product, the first exponential law is satisfied for any $n$-ary hyperalgebras $\mathbf{H}, \mathbf{K}$, not only for idempotent ones.

It may easily be seen that the direct product of a family of medial $n$-ary hyperalgebras is a medial $n$-ary hyperalgebra. But this is not generally true for the combined product of $n$-ary hyperalgebras. With respect to the combined product, we have the following weak form of the second exponential law:

Proposition 3.12. Let $\mathbf{G}_{i}, i \in I$, be a non-empty family of medial n-ary hyperalgebras and $\mathbf{H}$ be an n-ary hyperalgebra. If $\otimes_{i \in I} \mathbf{G}_{i}$ is medial, then

$$
\bigotimes_{i \in I} \mathbf{G}_{i}^{\mathbf{H}} \preceq\left(\bigotimes_{i \in I} \mathbf{G}_{i}\right)^{\mathbf{H}}
$$

Proof. Let $\mathbf{G}_{i}=\left(G_{i}, p^{i}\right)$ for every $i \in I, \mathbf{H}=(H, q), \bigotimes_{i \in I} \mathbf{G}_{i}=\left(\prod_{i \in I} G_{i}, r\right)$, $\mathbf{G}_{i}^{H}=\left(\operatorname{Hom}\left(\mathbf{H}, \mathbf{G}_{i}\right), u^{i}\right)$ for every $i \in I, \bigotimes_{i \in I} \mathbf{G}_{i}{ }^{\mathbf{H}}=\left(\prod_{i \in I} \operatorname{Hom}\left(\mathbf{H}, \mathbf{G}_{i}\right), s\right)$ and $\left(\bigotimes_{i \in I} \mathbf{G}_{i}\right)^{\mathbf{H}}=\left(\operatorname{Hom}\left(\mathbf{H}, \boldsymbol{\bigotimes}_{i \in I} \mathbf{G}_{i}\right), t\right)$. We define a map $\alpha: \prod_{i \in I} \operatorname{Hom}\left(\mathbf{H}, \mathbf{G}_{i}\right) \rightarrow$ $\left(\prod_{i \in I} G_{i}\right)^{H}$ by $\alpha\left(f^{i} ; i \in I\right)(z)=\left(f^{i}(z) ; i \in I\right)$ for each $z \in H$.

Let $\left(f^{i} ; i \in I\right) \in \prod_{i \in I} \operatorname{Hom}\left(\mathbf{H}, \mathbf{G}_{i}\right)$. Let $h \in \alpha\left(f^{i} ; i \in I\right)\left(q\left(x_{1}, \ldots, x_{n}\right)\right)$. Then, $h=\alpha\left(f^{i} ; i \in I\right)(x)$ where $x \in q\left(x_{1}, \ldots, x_{n}\right)$. Thus, $h=\alpha\left(f^{i} ; i \in I\right)(x)=$ $\left(f^{i}(x) ; i \in I\right)$. Since $x \in q\left(x_{1}, \ldots, x_{n}\right)$ and $f^{i} \in \operatorname{Hom}\left(\mathbf{H}, \mathbf{G}_{i}\right)$ for every $i \in I$, we have $f^{i}(x) \in p^{i}\left(f^{i}\left(x_{1}\right), \ldots, f^{i}\left(x_{n}\right)\right)$ for every $i \in I$. Thus, $h=\left(f^{i}(x) ; i \in I\right) \in$ $r\left(\left(f^{i}\left(x_{1}\right) ; i \in I\right), \ldots,\left(f^{i}\left(x_{n}\right) ; i \in I\right)\right)=r\left(\alpha\left(f^{i} ; i \in I\right)\left(x_{1}\right), \ldots, \alpha\left(f^{i} ; i \in I\right)\left(x_{n}\right)\right)$. Consequently, $\alpha\left(f^{i} ; i \in I\right)\left(q\left(x_{1}, \ldots, x_{n}\right)\right) \subseteq r\left(\alpha\left(f^{i} ; i \in I\right)\left(x_{1}\right), \ldots, \alpha\left(f^{i} ; i \in\right.\right.$ $\left.I)\left(x_{n}\right)\right)$. Therefore, $\alpha\left(f^{i} ; i \in I\right) \in \operatorname{Hom}\left(\mathbf{H}, \bigotimes_{i \in I} \mathbf{G}_{i}\right)$. We have shown that $\alpha$ maps $\prod_{i \in I} \operatorname{Hom}\left(\mathbf{H}, \mathbf{G}_{i}\right)$ into $\operatorname{Hom}\left(\mathbf{H}, \bigotimes_{i \in I} \mathbf{G}_{i}\right)$.

Suppose that $\alpha\left(f^{i} ; i \in I\right)=\alpha\left(g^{i} ; i \in I\right)$ where $\left(f^{i} ; i \in I\right),\left(g^{i} ; i \in I\right) \in$ $\prod_{i \in I} \operatorname{Hom}\left(\mathbf{H}, \mathbf{G}_{i}\right)$. Then $\left(f^{i}(x) ; i \in I\right)=\alpha\left(f^{i} ; i \in I\right)(x)=\alpha\left(g^{i} ; i \in I\right)(x)=$ $\left(g^{i}(x) ; i \in I\right)$ for every $x \in H$. Therefore, $f^{i}(x)=g^{i}(x)$ for every $i \in I$ and for every $x \in H$. Hence, $f^{i}=g^{i}$ for every $i \in I$. Thus, $\alpha$ : $\prod_{i \in I} \operatorname{Hom}\left(\mathbf{H}, \mathbf{G}_{i}\right) \rightarrow$ $\operatorname{Hom}\left(\mathbf{H}, \bigotimes_{i \in I} \mathbf{G}_{i}\right)$ is an injection.

Finally, let $\left(f_{1}^{i} ; i \in I\right), \ldots,\left(f_{n}^{i} ; i \in I\right) \in \prod_{i \in I} \operatorname{Hom}\left(\mathbf{H}, \mathbf{G}_{i}\right)$. We are to show that $\alpha\left(s\left(\left(f_{1}^{i} ; i \in I\right), \ldots,\left(f_{n}^{i} ; i \in I\right)\right)\right)=t\left(\alpha\left(f_{1}^{i} ; i \in I\right), \ldots, \alpha\left(f_{n}^{i} ; i \in I\right)\right)$. It is easy to see that the following seven conditions are equivalent:

(a) $f \in \alpha\left(s_{\lambda}\left(\left(f_{1}^{i} ; i \in I\right), \ldots,\left(f_{n}^{i} ; i \in I\right)\right)\right)$;

(b) $f=\alpha\left(f^{i} ; i \in I\right)$ where $\left(f^{i} ; i \in I\right) \in s\left(\left(f_{1}^{i} ; i \in I\right), \ldots,\left(f_{n}^{i} ; i \in I\right)\right)$;

(c) $f=\alpha\left(f^{i} ; i \in I\right)$ and there exists $J \subseteq I$, card $J \leq 1$, such that $f^{i} \in$ $u^{i}\left(f_{1}^{i}, \ldots, f_{n}^{i}\right)$ for every $i \in I \backslash J$ and $f^{i}=f_{1}^{i}=f_{2}^{i}=\ldots=f_{n}^{i}$ for every $i \in J$

(d) $f(x)=\left(f^{i}(x) ; i \in I\right)$ for every $x \in H$ and there exists $J \subseteq I$, card $J \leq 1$, such that $f^{i}(x) \in p_{\lambda}^{i}\left(f_{1}^{i}(x), \ldots, f_{n}^{i}(x)\right)$ for every $i \in I \backslash J$ and every $x \in H$ and $f^{i}(x)=f_{1}^{i}(x)=f_{2}^{i}(x)=\ldots=f_{n}^{i}(x)$ for every $i \in J$ and every $x \in H$;

(e) $f(x)=\left(f^{i}(x) ; i \in I\right) \in r\left(\left(f_{1}^{i}(x) ; i \in I\right), \ldots,\left(f_{n}^{i}(x) ; i \in I\right)\right)$ for every $x \in H$

(f) $f(x) \in r\left(\alpha\left(f_{1}^{i} ; i \in I\right)(x), \ldots, \alpha\left(f_{n}^{i} ; i \in I\right)(x)\right)$ for every $x \in H$;

(g) $f \in t\left(\alpha\left(f_{1}^{i} ; i \in I\right), \ldots, \alpha\left(f_{n}^{i} ; i \in I\right)\right)$. 
Consequently, $\alpha\left(s\left(\left(f_{1}^{i} ; i \in I\right), \ldots,\left(f_{n}^{i} ; i \in I\right)\right)\right)=t\left(\alpha\left(f_{1}^{i} ; i \in I\right), \ldots, \alpha\left(f_{n}^{i} ; i \in I\right)\right)$, which yields $\bigotimes_{i \in I} \mathbf{G}_{i}^{\mathbf{H}} \preceq\left(\bigotimes_{i \in I} \mathbf{G}_{i}\right)^{\mathbf{H}}$.

Remark 3.13. It may easily be shown that, in the previous Proposition, we may write $\cong$ instead of $\preceq$ provided that $\mathbf{G}_{i}$ is idempotent for every $i \in I$. We then obtain the second exponential law for the direct product, i.e., the law $\prod_{i \in I} \mathbf{G}_{i}^{\mathbf{H}} \cong$ $\left(\prod_{i \in I} \mathbf{G}_{i}\right)^{\mathbf{H}}$. We leave it as an open problem to find a necessary and sufficient condition for the validity of the second exponential law for the direct power of $n$ ary hyperalgebras with respect to the combined product, i.e., the law $\bigotimes_{i \in I} \mathbf{G}_{i}^{\mathbf{H}} \cong$ $\left(\bigotimes_{i \in I} \mathbf{G}_{i}\right)^{\mathbf{H}}$.

\section{REFERENCES}

[1] G. Birkhoff, Generalized arithmetic, Duke Math. J. 9 (1942), 283-302. DOI: 10.1215/S00127094-42-00921-9

[2] P. Corsini (ed.), Algebraic Hyperstructures and Applications, Proceedinds of the Sixth International Congress, Prague, 1996, Democritus University of Thrace, Greece, 1997.

[3] J. Ježek and T. Kepka, Medial Groupoids, Rozpravy ČSAV, Řada Mat. a Př́r. Věd. 93/1, Academia, Prague, 1983.

[4] L. Klukovits, On commutative universal algebras, Acta Sci. Math. (Szeged) 34 (1973), 171174.

[5] F. E. Linton, Autonomous equational categories, J. Math. Mech. 15 (1966), 637-642.

[6] V. Novák, On a power of relational structures, Czech. Math. J. 35 (1985), 321-328.

[7] M. Novotný and J. Šlapal, On powers of relational structures, Publ. Math. Debrecen 61 (2002), 341-356.

[8] J. Plonka, Diagonal algebras, Fund. Math. 58 (1966), 309-321.

[9] J. Šlapal, Direct arithmetic of relational systems, Publ. Math. Debrecen 38 (1991), 39-48.

[10] J. Šlapal, Cardinal arithmetic of general relational systems, Czech. Math. J. 43 (1993), $125-139$.

[11] J. Šlapal, On exponentiation of n-ary algebras, Acta Math. Hungar. 63 (1994), 313-322.

[12] J. Šlapal, On exponentiation of universal hyperalgebras, Alg. Universalis 44 (2000), 187-193.

[13] J. Šlapal, Exponentiality in categories of partial algebras, Mat. Pannon. 13 (2002), 201-206.

Nitima Chaisansuk, Department of Mathematics, Chiang Mai University, Faculty of Science, 239 Huaykaew Road, Tumbol Suthep Amphur Muang, 50200 Chiang Mai, Thailand e-mail: nitimachaisansuk@gmail.com 